Vietnam Journal of Mechanics, VAST, Vol.38, No. 2 (2016), pp. 123-140

DOI:10.15625/0866-7136/38/2/7066

\title{
POSTBUCKLING OF THICK FGM CYLINDRICAL PANELS WITH TANGENTIAL EDGE CONSTRAINTS AND TEMPERATURE DEPENDENT PROPERTIES
}

\author{
Hoang Van Tung \\ Hanoi Architectural University, Vietnam \\ E-mail: inter0105@gmail.com \\ Received September 21, 2015
}

\begin{abstract}
This paper investigates postbuckling behavior of thick FGM cylindrical panels resting on elastic foundations and subjected to thermal, mechanical and thermomechanical loading conditions. Material properties are assumed to be temperature dependent, and graded in the thickness direction according to a simple power law distribution in terms of the volume fractions of constituents. Governing equations are based on higher order shear deformation shell theory incorporating von Karman-Donnell geometrical nonlinearity, initial geometrical imperfection, tangential edge constraints and Pasternak type elastic foundations. Approximate solutions are assumed to satisfy simply supported boundary conditions and Galerkin procedure is applied to derive expressions of buckling loads and load-deflection relations. In thermal postbuckling analysis, an iteration algorithm is employed to determine critical buckling temperatures and postbuckling temperaturedeflection equilibrium paths. The separate and simultaneous effects of tangential edge restraints, elastic foundations and temperature dependence of material properties on the buckling and postbuckling responses of higher order shear deformable FGM cylindrical panels are analyzed and discussed.
\end{abstract}

Keywords: Functionally graded materials, tangential edge constraint, cylindrical panel, buckling and postbuckling, elastic foundations.

\section{INTRODUCTION}

Structural elements in form of cylindrical panels are widely used as major portions in the construction of aircraft, missile and aerospace vehicle. Cylindrical panels also find their important applications in building construction, underground structures and shipbuilding industry. Since these panels are frequently subjected to severe loading conditions, their static and dynamic responses are governing problems for accurate predictions and safe design. The appearance of functionally graded material (FGM) with advanced and novel features necessitates more studies on the behavior of FGM structures in general, and the stability of FGM cylindrical panels in particular. As a result,

(C) 2016 Vietnam Academy of Science and Technology 
buckling and postbuckling of FGM cylindrical panels are attractive topics for many researchers. Nonlinear response and postbuckling behavior of thin and moderately thick FGM cylindrical panels subjected to mechanical and thermomechanical loads have been investigated by Woo et al. [1], Duc and Tung [2-4] using analytical approaches. Basing on asymptotic solutions and an iteration algorithm, Shen and his co-workers have analyzed nonlinear bending and postbuckling of FGM cylindrical panels under mechanical, thermal and electric loadings [5-7]. Geometrically nonlinear response of FGM cylindrical panels under external pressure and axial compression has been addressed by Liew and his collaborators [8-10] using meshless methods. Recently, Shen and Wang [8-10] using meshless methods. Recently, Shen and Wang [11,12] studied the effects of elastic foundations on the nonlinear bending and postbuckling of FGM cylindrical panels utilizing a semi-analytical approach. Effects of stiffeners on the nonlinear static and dynamic responses of thin FGM cylindrical and doubly curved panels without elastic foundations have been analyzed by Bich et al. $[13,14]$ on the basis of thin shell theory. These works are then extended by Duc and Quan $[15,16]$ for case of thin and curved FGM panels resting on elastic foundations and exposed to thermal environments.

In foregoing studies, only two extreme cases of in-plane constraints of edges have been considered. Specifically, the edges of panels are usually assumed to be either unrestrained (movable edges) or fully restrained (immovable edges). However, in practical situations, the tangential motion of the edges may be partially restrained only. As mentioned by Librescu et al. $[17,18]$, tangential edge constraints have great and important effects on the nonlinear behavior of laminated composite plate and shells. Recently, Tung used an analytical approach based on the classical shell theory to analyze the postbuckling behavior of thin FGM cylindrical panels and circular cylindrical shells subjected to mechanical and thermal loads taking the effects of tangential edge constraints into consideration $[19,20]$ without and with temperature dependent material properties, respectively.

This paper extends previous works $[4,19]$ to investigate the postbuckling behavior of higher order shear deformable FGM cylindrical panels resting on elastic foundations and subjected to mechanical, thermal and thermomechanical loads. New contributions of the present work are that the separate and combined effects of various degrees of tangential edge constraints and temperature dependence of material properties on the postbuckling behavior of thick FGM cylindrical panels are taken into consideration.

\section{FGM CYLINDRICAL PANEL ON AN ELASTICAL FOUNDATION}

Consider an FGM cylindrical panel with radius of curvature $R$, thickness $h$, axial length $a$ and arc length $b$ resting on a Pasternak type elastic foundation as shown in Fig. 1 .

The panel is made from a mixture of ceramics and metals, and is defined in a coordinate system $(x, y, z)$ whose origin is located at the corner on the middle surface of the shell panel, $x$ and $y$ are in the axial and circumferential directions, respectively, and $z$ is perpendicular to the middle surface and points inwards $(-h / 2 \leq z \leq h / 2)$.

Suppose that the material composition of the shell panel varies smoothly along the thickness in such a way that the inner surface is metal-rich and the outer surface is ceramic-rich by following a simple power law in terms of the volume fractions of the 


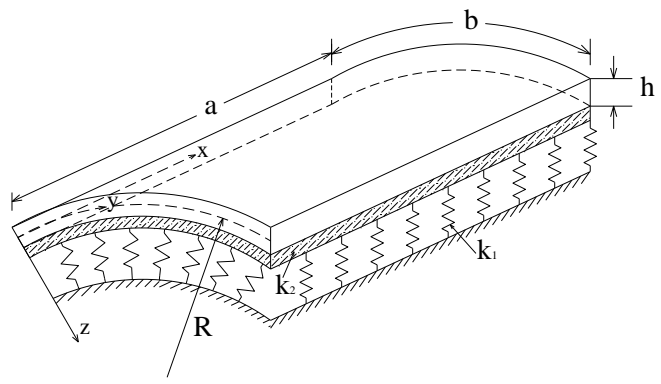

Fig. 1. Configuration and coordinate system of a cylindrical panel on a Pasternak elastic foundation

constituents as

$$
V_{m}(z)=\left(\frac{2 z+h}{2 h}\right)^{N}, \quad V_{c}(z)=1-V_{m}(z)
$$

where $V_{m}$ and $V_{c}$ are the volume fractions of metal and ceramic constituents, respectively, and $N \geq 0$ is volume fraction index.

Practically, FGMs are most commonly used in high temperature environments, and significant changes in material properties are inherent. Usually, the elasticity modulus decreases, and the thermal expansion coefficient increases at elevated temperatures. Therefore, it is essential to account for this temperature dependence for accurate and reliable prediction of the response of FGM cylindrical panels in thermal environments.

It is assumed that the effective properties $\operatorname{Pr}_{\text {eff }}$ of FGM cylindrical panels change only in the thickness direction $z$ and can be determined by the linear rule of mixture as

$$
\operatorname{Pr}_{\text {eff }}(z, T)=\operatorname{Pr}_{m}(T) V_{m}(z)+\operatorname{Pr}_{c}(T) V_{c}(z),
$$

where Pr denotes a specific material property assumed to be temperature-dependent in the present study, and subscripts $m$ and $c$ represent the metal and ceramic constituents, respectively. From Eqs. (1) and (2) the effective properties of FGM panels such as the modulus of elasticity $E$ and the coefficient of thermal expansion $\alpha$ can be written as

$$
\begin{aligned}
& E(z, T)=E_{c}(T)+E_{m c}(T)\left(\frac{2 z+h}{2 h}\right)^{N}, \\
& \alpha(z, T)=\alpha_{c}(T)+\alpha_{m c}(T)\left(\frac{2 z+h}{2 h}\right)^{N}, \quad v(z, T)=v,
\end{aligned}
$$

where Poisson's ratio $v$ is assumed to be constant and

$$
E_{m c}(T)=E_{m}(T)-E_{c}(T), \quad \alpha_{m c}(T)=\alpha_{m}(T)-\alpha_{c}(T) .
$$

In the present study, the FGM cylindrical panel is fully rested on an elastic foundation and the FGM panel-foundation interaction is represented by two-parameter model as

$$
q_{f}=k_{1} w-k_{2} \Delta w,
$$

where $\Delta=\partial^{2} / \partial x^{2}+\partial^{2} / \partial y^{2}$ is Laplace operator, $w$ is the deflection (transverse displacement) of the panel, $k_{1}$ is Winkler foundation modulus and $k_{2}$ is the shear layer foundation stiffness of Pasternak model. 


\section{THEORETICAL FORMULATIONS}

In the present study, higher order shear deformation shell theory developed by Reddy and Liu [21] is used to establish governing equations and derive expressions for nonlinear response analysis of thick FGM cylindrical panels. Based on this theory, normal strains $\varepsilon_{x}, \varepsilon_{y}$, in-plane shear strain $\gamma_{x y}$ and transverse shear deformations $\gamma_{x z}, \gamma_{y z}$ are represented as

$$
\left(\begin{array}{c}
\varepsilon_{x} \\
\varepsilon_{y} \\
\gamma_{x y}
\end{array}\right)=\left(\begin{array}{c}
\varepsilon_{x}^{0} \\
\varepsilon_{y}^{0} \\
\gamma_{x y}^{0}
\end{array}\right)+z\left(\begin{array}{c}
k_{x}^{1} \\
k_{y}^{1} \\
k_{x y}^{1}
\end{array}\right)+z^{3}\left(\begin{array}{c}
k_{x}^{3} \\
k_{y}^{3} \\
k_{x y}^{3}
\end{array}\right), \quad\left(\begin{array}{c}
\gamma_{x z} \\
\gamma_{y z}
\end{array}\right)=\left(\begin{array}{c}
\gamma_{x z}^{0} \\
\gamma_{y z}^{0}
\end{array}\right)+z^{2}\left(\begin{array}{c}
k_{x z}^{2} \\
k_{y z}^{2}
\end{array}\right),
$$

where

$$
\begin{aligned}
& \left(\begin{array}{c}
\varepsilon_{x}^{0} \\
\varepsilon_{y}^{0} \\
\gamma_{x y}^{0}
\end{array}\right)=\left(\begin{array}{c}
u_{, x}+w_{, x}^{2} / 2 \\
v_{, y}-w / R+w_{, y}^{2} / 2 \\
u_{, y}+v_{, x}+w_{, x} w_{, y}
\end{array}\right), \quad\left(\begin{array}{c}
k_{x}^{1} \\
k_{y}^{1} \\
k_{x y}^{1}
\end{array}\right)=\left(\begin{array}{c}
\phi_{x, x} \\
\phi_{y, y} \\
\phi_{x, y}+\phi_{y, x}
\end{array}\right), \\
& \left(\begin{array}{c}
k_{x}^{3} \\
k_{y}^{3} \\
k_{x y}^{3}
\end{array}\right)=-c_{1}\left(\begin{array}{c}
\phi_{y, y}+w_{, x x} \\
\phi_{x, y}+\phi_{y, x}+2 w_{, x y}
\end{array}\right), \\
& \left(\begin{array}{c}
\gamma_{x z}^{0} \\
\gamma_{y z}^{0}
\end{array}\right)=\left(\begin{array}{c}
\phi_{x}+w_{, x} \\
\phi_{y}+w_{, y}
\end{array}\right), \quad\left(\begin{array}{c}
k_{x z}^{2} \\
k_{y z}^{2}
\end{array}\right)=-3 c_{1}\left(\begin{array}{c}
\phi_{x}+w_{, x}+w_{, x}^{*} \\
\phi_{y}+w_{, y}+w_{, y}^{*}
\end{array}\right),
\end{aligned}
$$

in which $c_{1}=4 /\left(3 h^{2}\right)$ and von Karman nonlinear terms are incorporated. Also, $u, v$ are displacement components along the $x, y$ directions, respectively, and $\phi_{x}, \phi_{y}$ are the rotations of normal to the midsurface with respect to $y$ and $x$ axes, respectively. Moreover, $w^{*}$ is a known function representing initial geometrical imperfection of the cylindrical panel.

Hookes law for an FGM cylindrical panel is defined as

$$
\begin{aligned}
& \sigma_{x}=\frac{E(z, T)}{1-v^{2}}\left[\varepsilon_{x}+v \varepsilon_{y}-(1+v) \alpha(z, T) \Delta T\right], \\
& \sigma_{y}=\frac{E(z, T)}{1-v^{2}}\left[\varepsilon_{y}+v \varepsilon_{x}-(1+v) \alpha(z, T) \Delta T\right], \\
& {\left[\sigma_{x y}, \sigma_{x z}, \sigma_{y z}\right]=\frac{E(z, T)}{2(1+v)}\left[\gamma_{x y}, \gamma_{x z}, \gamma_{y z}\right],}
\end{aligned}
$$

where $\Delta T$ is temperature rise from thermal stress-free initial state, and is assumed to be independent of in-plane coordinates $x, y$. The force and moment resultants of the FGM panel are determined as

$$
\begin{aligned}
& \left(N_{i}, M_{i}, P_{i}\right)=\int_{-h / 2}^{h / 2} \sigma_{i}\left(1, z, z^{3}\right) d z, \quad i=x, y, x y \\
& \left(Q_{x}, K_{x}\right)=\int_{-h / 2}^{h / 2} \sigma_{x z}\left(1, z^{2}\right) d z, \quad\left(Q_{y}, K_{y}\right)=\int_{-h / 2}^{h / 2} \sigma_{y z}\left(1, z^{2}\right) d z .
\end{aligned}
$$


Introduction of Eqs. (6), (7) into Eqs. (8) and substitution of the resulting into Eqs. (9) give the constitutive relations as

$$
\begin{aligned}
& \left(N_{x}, M_{x}, P_{x}\right)=\frac{1}{1-v^{2}}\left[\left(E_{1}, E_{2}, E_{4}\right)\left(\varepsilon_{x}^{0}+v \varepsilon_{y}^{0}\right)+\left(E_{2}, E_{3}, E_{5}\right)\left(k_{x}^{1}+v k_{y}^{1}\right)\right. \\
& \left.+\left(E_{4}, E_{5}, E_{7}\right)\left(k_{x}^{3}+v k_{y}^{3}\right)-(1+v)\left(\Phi_{1}, \Phi_{2}, \Phi_{4}\right)\right], \\
& \left(N_{y}, M_{y}, P_{y}\right)=\frac{1}{1-v^{2}}\left[\left(E_{1}, E_{2}, E_{4}\right)\left(\varepsilon_{y}^{0}+v \varepsilon_{x}^{0}\right)+\left(E_{2}, E_{3}, E_{5}\right)\left(k_{y}^{1}+v k_{x}^{1}\right)\right. \\
& \left.+\left(E_{4}, E_{5}, E_{7}\right)\left(k_{y}^{3}+v k_{x}^{3}\right)-(1+v)\left(\Phi_{1}, \Phi_{2}, \Phi_{4}\right)\right], \\
& \left(N_{x y}, M_{x y}, P_{x y}\right)=\frac{1}{2(1+v)}\left[\left(E_{1}, E_{2}, E_{4}\right) \gamma_{x y}^{0}+\left(E_{2}, E_{3}, E_{5}\right) k_{x y}^{1}+\left(E_{4}, E_{5}, E_{7}\right) k_{x y}^{3}\right], \\
& \left(Q_{x}, K_{x}\right)=\frac{1}{2(1+v)}\left[\left(E_{1}, E_{3}\right) \gamma_{x z}^{0}+\left(E_{3}, E_{5}\right) k_{x z}^{2}\right] \text {, } \\
& \left(Q_{y}, K_{y}\right)=\frac{1}{2(1+v)}\left[\left(E_{1}, E_{3}\right) \gamma_{y z}^{0}+\left(E_{3}, E_{5}\right) k_{y z}^{2}\right] \text {, }
\end{aligned}
$$

where

$$
\begin{aligned}
& \left(E_{1}, E_{2}, E_{3}, E_{4}, E_{5}, E_{7}\right)=\int_{-h / 2}^{h / 2} E(z, T)\left(1, z, z^{2}, z^{3}, z^{4}, z^{6}\right) d z, \\
& \left(\Phi_{1}, \Phi_{2}, \Phi_{4}\right)=\int_{-h / 2}^{h / 2} E(z, T) \alpha(z, T) \Delta T\left(1, z, z^{3}\right) d z,
\end{aligned}
$$

and specific expressions of coefficients $E_{i}=E_{i}(T)(i=1 \div 7)$ are analogous to those given in [4] for case of temperature independent properties and omitted here for sake of brevity. Nonlinear equilibrium equation of an imperfect FGM cylindrical panel on an elastic foundation on the basis of higher order shear deformation shell theory has the form [4]

$$
\begin{aligned}
c_{1}^{2} & \left(D_{2} D_{5} / D_{4}-D_{3}\right) \Delta^{3} w+\left(c_{1} D_{2} / D_{4}+1\right) D_{6} \Delta^{2} w \\
& +\left(1-c_{1} D_{5} / D_{4}\right) \Delta\left[f_{, y y}\left(w_{, x x}+w_{, x x}^{*}\right)-2 f_{, x y}\left(w_{, x y}+w_{, x y}^{*}\right)\right. \\
& \left.+f_{, x x}\left(w_{, y y}+w_{, y y}^{*}\right)+f_{, x x} / R+q-k_{1} w+k_{2} \Delta w\right]-D_{6} / D_{4}\left[f_{, y y}\left(w_{, x x}+w_{, x x}^{*}\right)\right. \\
& \left.-2 f_{, x y}\left(w_{, x y}+w_{, x y}^{*}\right)+f_{, x x}\left(w_{, y y}+w_{, y y}^{*}\right)+f_{, x x} / R+q-k_{1} w+k_{2} \Delta w\right]=0,
\end{aligned}
$$

where $q$ is external pressure uniformly distributed on the upper surface of the panel, $f(x, y)$ is a stress function defined as

$$
N_{x}=f_{, y y}, \quad N_{y}=f_{, x x}, \quad N_{x y}=-f_{, x y},
$$


and

$$
\begin{aligned}
& D_{1}=\frac{E_{1} E_{3}-E_{2}^{2}}{E_{1}\left(1-v^{2}\right)}, \quad D_{2}=\frac{E_{1} E_{5}-E_{2} E_{4}}{E_{1}\left(1-v^{2}\right)}, \quad D_{3}=\frac{E_{1} E_{7}-E_{4}^{2}}{E_{1}\left(1-v^{2}\right)^{\prime}} \\
& D_{4}=D_{1}-c_{1} D_{2}, \quad D_{5}=D_{2}-c_{1} D_{3}, \quad D_{6}=\frac{1}{2(1+v)}\left(E_{1}-6 c_{1} E_{3}+9 c_{1}^{2} E_{5}\right) .
\end{aligned}
$$

Strain compatibility equation for an imperfect FGM cylindrical panel is written as $[2,4]$

$$
\Delta^{2} f-E_{1}\left(w_{, x y}^{2}-w_{, x x} w_{, y y}+2 w_{, x y} w_{, x y}^{*}-w_{, x x} w_{, y y}^{*}-w_{, y y} w_{, x x}^{*}-w_{, x x} / R\right)=0 .
$$

Eqs. (12) and (15) are governing equations for postbuckling analysis of FGM panels.

In this study, the FGM cylindrical panels are assumed to be simply supported at all edges. The associated boundary conditions are $[4,21]$

$$
\begin{aligned}
& w=\phi_{y}=M_{x}=P_{x}=0, N_{x}=N_{x 0} \text { at } x=0, a \\
& w=\phi_{x}=M_{y}=P_{y}=0, N_{y}=N_{y 0} \text { at } y=0, b
\end{aligned}
$$

In Eqs. (16), $N_{x 0}, N_{y 0}$ are prebuckling compressive force resultants at freely movable edges where $N_{x y}$ is zero-valued, and are fictitious compressive edge loads rendering the edges partially movable or immovable.

For the purpose of the present study, in-plane boundary conditions are assumed to be with varying degrees of tangential edge restraint. The degree of tangential edge restraint considered is bounded by the cases in which the tangential motion of the unloaded edges of a panel are either unrestrained or completely restrained, respectively, in the in-plane direction perpendicular to the panel edge. For these two cases, the panel edges are referred to as movable and immovable edges, respectively. All intermediate cases are referred to herein as partially movable edges and include elastically restrained edge constraints.

The average end-shortening displacement $\Delta_{1}$ between edges $x=0$ and $x=a$ is related to the corresponding fictitious compressive edge load $N_{x 0}$ by

$$
\Delta_{1} s_{1}=N_{x 0}
$$

where $s_{1}$ is the average tangential stiffness in the $x$ direction on each opposite edge. Similarly, for the edges $y=0$ and $y=b$, relation is expressed as

$$
\Delta_{2} s_{2}=N_{y 0},
$$

where $s_{2}$ is the average tangential stiffness in the $y$ direction on each opposite edge.

The expressions for the average end-shortening displacements are defined as [17, 18]

$$
\Delta_{1}=-\frac{1}{a b} \int_{0}^{a} \int_{0}^{b} \frac{\partial u}{\partial x} d y d x, \quad \Delta_{2}=-\frac{1}{a b} \int_{0}^{a} \int_{0}^{b} \frac{\partial v}{\partial y} d y d x .
$$

Eqs. (17) and (18) indicate that values of $\Delta_{1}=0$ and $\Delta_{2}=0$ correspond to immovable edges at $x=0, a$ and $y=0, b$, respectively. These conditions are satisfied by selecting $s_{1} \rightarrow \infty$ and $s_{2} \rightarrow \infty$, respectively. In addition, values of $s_{1}=0$ and $s_{2}=0$ correspond to 
movable edges at $x=0, a$ and $y=0, b$, respectively. For these movable edge conditions, fictitious compressive edge loads are zero-valued, i.e. $N_{x 0}=0$ and $N_{y 0}=0$.

To satisfy boundary conditions (16), the approximate solutions are assumed as [4]

$$
\begin{aligned}
& \left(w, w^{*}\right)=(W, \mu h) \sin \beta_{m} x \sin \delta_{n} y, \\
& f=A_{1} \cos 2 \beta_{m} x+A_{2} \cos 2 \delta_{n} y+A_{3} \sin \beta_{m} x \sin \delta_{n} y+\frac{1}{2} N_{x 0} y^{2}+\frac{1}{2} N_{y 0} x^{2}, \\
& \phi_{x}=B_{1} \cos \beta_{m} x \sin \delta_{n} y, \quad \phi_{y}=B_{2} \sin \beta_{m} x \cos \delta_{n} y,
\end{aligned}
$$

where $\beta_{m}=m \pi / a, \delta_{n}=n \pi / b, W$ is amplitude of the deflection and $\mu$ is imperfection parameter. The coefficients $A_{i}(i=1 \div 3)$ are determined by substitution of Eqs. (20) and (21) into Eq. (15) as

$$
A_{1}=\frac{E_{1} \delta_{n}^{2}}{32 \beta_{m}^{2}} W(W+2 \mu h), \quad A_{2}=\frac{E_{1} \beta_{m}^{2}}{32 \delta_{n}^{2}} W(W+2 \mu h), \quad A_{3}=\frac{E_{1} \beta_{m}^{2}}{\left(\beta_{m}^{2}+\delta_{n}^{2}\right)^{2} R} W,
$$

and detail of coefficients $B_{1}, B_{2}$ in Eq. (22) are given in the work [4].

Subsequently, Eqs. (20), (21) are substituted into equilibrium equation (12) and applying Galerkin method for the resulting equation as procedure developed in the [4] yield

$$
\begin{aligned}
& \left\{-\frac{\left(\bar{D}_{2} \bar{D}_{5}-\bar{D}_{3} \bar{D}_{4}\right)}{9 \bar{D}_{6} B_{h}^{6}} m n \pi^{8}\left(m^{2} B_{a}^{2}+n^{2}\right)^{3}+\frac{m n \pi^{6}}{16 B_{h}^{4}}\left(\frac{4}{3} \bar{D}_{2}+\bar{D}_{4}\right)\left(m^{2} B_{a}^{2}+n^{2}\right)^{2}\right. \\
& \left.+\frac{\bar{E}_{1} m^{5} n \pi^{2} B_{a}^{6} R_{a}^{2}}{16 B_{h}^{2}\left(m^{2} B_{a}^{2}+n^{2}\right)^{2}} \bar{\xi}_{m n}+\frac{m n \pi^{2} \bar{D}_{1} B_{a}^{2}}{16 B_{h}^{4}} \bar{\xi}_{m n}\left[B_{a}^{2} K_{1}+\pi^{2}\left(m^{2} B_{a}^{2}+n^{2}\right) K_{2}\right]\right\} \bar{W} \\
& -\frac{2 m^{4} n^{2} \pi^{2} \bar{E}_{1} B_{a}^{5} R_{a}}{3 B_{h}^{3}\left(m^{2} B_{a}^{2}+n^{2}\right)^{2}}\left(2 \bar{\xi}_{m n}-1\right) \bar{W}(\bar{W}+\mu) \\
& -\frac{\bar{E}_{1} n^{2} \pi^{2} B_{a} R_{a}}{24 B_{h}^{3}}\left[\frac{4 \pi^{2} m^{2} B_{a}^{2}}{3 \bar{D}_{6} B_{h}^{2}}\left(3 \bar{D}_{4}-4 \bar{D}_{5}\right)+1\right] \bar{W}(\bar{W}+2 \mu) \\
& +\frac{\bar{E}_{1} m n \pi^{6}}{256 B_{h}^{4}}\left(m^{4} B_{a}^{4}+n^{4}\right) \bar{\xi}_{m n} \bar{W}(\bar{W}+\mu)(\bar{W}+2 \mu) \\
& +\frac{m n \pi^{4}}{16 B_{h}^{2}} \bar{\xi}_{m n}\left(m^{2} B_{a}^{2} \bar{N}_{x 0}+n^{2} \bar{N}_{y 0}\right)(\bar{W}+\mu)-\frac{B_{a} R_{a}}{B_{h}} \bar{N}_{y 0}-q=0,
\end{aligned}
$$

where $m, n$ are odd numbers, and

$$
\begin{aligned}
& \bar{E}_{i}=E_{i} / h^{i}(i=1 \div 7), \bar{W}=W / h, B_{a}=b / a, B_{h}=b / h, R_{a}=a / R, \bar{D}_{1}=D_{1} / h^{3}, \\
& \bar{D}_{2}=D_{2} / h^{5}, \bar{D}_{3}=D_{3} / h^{7}, \bar{D}_{4}=D_{4} / h^{3}, \bar{D}_{5}=D_{5} / h^{5}, \bar{D}_{6}=D_{6} / h, \bar{N}_{x 0}=N_{x 0} / h, \\
& \bar{N}_{y 0}=N_{y 0} / h, K_{1}=\frac{k_{1} a^{4}}{D_{1}}, K_{2}=\frac{k_{2} a^{2}}{D_{1}}, \bar{\zeta}_{m n}=\frac{\left(3 \bar{D}_{4}-4 \bar{D}_{5}\right) \pi^{2}}{3 \bar{D}_{6} B_{h}^{2}}\left(m^{2} B_{a}^{2}+n^{2}\right)+1 .
\end{aligned}
$$

In what follows, the fictitious compressive edge loads $N_{x 0}, N_{y 0}$ for the FGM panels under the tangential edge constraints will be specified. From Eqs. (6), (7) and (10) one 
can obtain the following relations in which Eq. (13) and imperfection have been included

$$
\begin{aligned}
& \frac{\partial u}{\partial x}=\frac{1}{E_{1}}\left(f_{, y y}-v f_{, x x}\right)-\frac{E_{2}}{E_{1}} \phi_{x, x}+\frac{c_{1} E_{4}}{E_{1}}\left(\phi_{x, x}+w_{, x x}\right)-\frac{1}{2} w_{, x}^{2}-w_{, x} w_{, x}^{*}+\frac{\Phi_{1}}{E_{1}}, \\
& \frac{\partial v}{\partial y}=\frac{1}{E_{1}}\left(f_{, x x}-v f_{, y y}\right)-\frac{E_{2}}{E_{1}} \phi_{y, y}+\frac{c_{1} E_{4}}{E_{1}}\left(\phi_{y, y}+w_{, y y}\right)-\frac{1}{2} w_{, y}^{2}-w_{, y} w_{, y}^{*}+\frac{\Phi_{1}}{E_{1}}+\frac{w}{R} .
\end{aligned}
$$

Introduction of Eqs. (20), (21) and (22) into Eqs. (26) and then substitution of the resulting equations into Eqs. (19) yield the following expressions

$$
\begin{aligned}
\Delta_{1}= & \frac{1}{E_{1}}\left(v N_{y 0}-N_{x 0}\right)+\frac{4\left(\delta_{n}^{2}-v \beta_{m}^{2}\right) \beta_{m}^{2}}{m n \pi^{2} R\left(\beta_{m}^{2}+\delta_{n}^{2}\right)^{2}} W-\frac{4 E_{2} B_{1} \beta_{m}}{m n \pi^{2} E_{1}} \\
& +\frac{4 c_{1} E_{4} \beta_{m}}{m n \pi^{2} E_{1}}\left(B_{1}+\beta_{m} W\right)+\frac{\beta_{m}^{2}}{8} W(W+2 \mu h)-\frac{\Phi_{1}}{E_{1}}, \\
\Delta_{2}= & \frac{1}{E_{1}}\left(v N_{x 0}-N_{y 0}\right)+\frac{4\left(\beta_{m}^{2}-v \delta_{n}^{2}\right) \beta_{m}^{2}}{m n \pi^{2} R\left(\beta_{m}^{2}+\delta_{n}^{2}\right)^{2}} W-\frac{4 E_{2} B_{2} \delta_{n}}{m n \pi^{2} E_{1}} \\
& +\frac{4 c_{1} E_{4} \delta_{n}}{m n \pi^{2} E_{1}}\left(B_{2}+\delta_{n} W\right)+\frac{\delta_{n}^{2}}{8} W(W+2 \mu h)-\frac{\Phi_{1}}{E_{1}}-\frac{4 W}{m n \pi^{2} R} .
\end{aligned}
$$

Now, introduction of Eqs. (27) into Eqs. (17), (18) and solving obtained equations, the fictitious compressive edge loads can be determined as

where

$$
\begin{aligned}
& \bar{N}_{x 0}=e_{11} \bar{W}+e_{12} \bar{W}(\bar{W}+2 \mu)-e_{13} \Phi_{1} / h, \\
& \bar{N}_{y 0}=e_{21} \bar{W}+e_{22} \bar{W}(\bar{W}+2 \mu)-e_{23} \Phi_{1} / h,
\end{aligned}
$$

$$
\begin{aligned}
e_{11}= & \frac{4 e \bar{E}_{1} m B_{a}^{3} R_{a}}{n \pi^{2} B_{h}\left(m^{2} B_{a}^{2}+n^{2}\right)^{2}}\left[\bar{s}_{1}\left(\bar{E}_{1}+\bar{s}_{2}\right)\left(n^{2}-v m^{2} B_{a}^{2}\right)+v \bar{s}_{1} \bar{s}_{2}\left(m^{2} B_{a}^{2}-v n^{2}\right)\right] \\
& -\frac{4 e \bar{E}_{2}}{m n \pi B_{h}}\left[\bar{s}_{1}\left(\bar{E}_{1}+\bar{s}_{2}\right) m B_{a} \bar{B}_{1}+v \bar{s}_{1} \bar{s}_{2} n \bar{B}_{2}\right]-\frac{4 v e \bar{s}_{1} \bar{s}_{2}}{m n \pi^{2} B_{h}} \bar{E}_{1} B_{a} R_{a} \\
& +\frac{16 e \bar{E}_{4}}{3 m n \pi B_{h}^{2}}\left[\bar{s}_{1}\left(\bar{E}_{1}+\bar{s}_{2}\right)\left(m B_{a} B_{h} \bar{B}_{1}+\pi m^{2} B_{a}^{2}\right)+v \bar{s}_{1} \bar{s}_{2}\left(n B_{h} \bar{B}_{2}+\pi n^{2}\right)\right], \\
e_{12}= & \frac{\pi^{2} e \bar{E}_{1}}{8 B_{h}^{2}}\left[\bar{s}_{1}\left(\bar{E}_{1}+\bar{s}_{2}\right) m^{2} B_{a}^{2}+v n^{2} \bar{s}_{1} \bar{s}_{2}\right], \quad e_{13}=e\left[v \bar{s}_{1} \bar{s}_{2}+\bar{s}_{1}\left(\bar{E}_{1}+\bar{s}_{2}\right)\right], \\
e_{21}= & \frac{4 e \bar{E}_{1} m B_{a}^{3} R_{a}}{n \pi^{2} B_{h}\left(m^{2} B_{a}^{2}+n^{2}\right)^{2}}\left[\bar{s}_{2}\left(\bar{E}_{1}+\bar{s}_{1}\right)\left(m^{2} B_{a}^{2}-v n^{2}\right)+v \bar{s}_{1} \bar{s}_{2}\left(n^{2}-v m^{2} B_{a}^{2}\right)\right] \\
& -\frac{4 e \bar{E}_{2}}{m n \pi B_{h}^{2}}\left[\bar{s}_{2}\left(\bar{E}_{1}+\bar{s}_{1}\right) n B_{h} \bar{B}_{2}+v \bar{s}_{1} \bar{s}_{2} m B_{a} B_{h} \bar{B}_{1}\right]-\frac{4 e \bar{s}_{2}\left(\bar{E}_{1}+\bar{s}_{1}\right)}{m n \pi^{2} B_{h}} \bar{E}_{1} B_{a} R_{a}, \\
& +\frac{16 e \bar{E}_{4}}{3 \pi m n B_{h}^{2}}\left[\bar{s}_{2}\left(\bar{E}_{1}+\bar{s}_{1}\right)\left(n B_{h} \bar{B}_{2}+\pi n^{2}\right)+v \bar{s}_{1} \bar{s}_{2}\left(m B_{a} B_{h} \bar{B}_{1}+\pi m^{2} B_{a}^{2}\right)\right], \\
e_{22}= & \frac{\pi^{2} e \bar{E}_{1}\left[v \bar{s}_{1} \bar{s}_{2} m^{2} B_{a}^{2}+\bar{s}_{2}\left(\bar{E}_{1}+\bar{s}_{1}\right) n^{2}\right], \quad e_{23}=e\left[v \bar{s}_{1} \bar{s}_{2}+\bar{s}_{2}\left(\bar{E}_{1}+\bar{s}_{1}\right)\right],}{8 B_{h}^{2}}
\end{aligned}
$$


in which the detail of $\bar{B}_{1}, \bar{B}_{2}$ can be found in the work [4], and

$$
\bar{s}_{1}=s_{1} / h, \quad \bar{s}_{2}=s_{2} / h, \quad e=\frac{1}{\left(\bar{E}_{1}+\bar{s}_{1}\right)\left(\bar{E}_{1}+\bar{s}_{2}\right)-v^{2} \bar{s}_{1} \bar{s}_{2}} .
$$

In this study, the FGM cylindrical panel is entirely exposed to thermal environments uniformly raised from thermal stress free initial state $T_{0}$ to value $T$ and temperature change $\Delta T=T-T_{0}$ is considered to be independent of thickness variable $z$. The thermal expression $\Phi_{1}$ is obtained from Eqs. (11) as

$$
\Phi_{1} / h=H(T) \Delta T, H(T)=E_{c}(T) \alpha_{c}(T)+\frac{E_{c}(T) \alpha_{m c}(T)+E_{m c}(T) \alpha_{c}(T)}{N+1}+\frac{E_{m c}(T) \alpha_{m c}(T)}{2 N+1} .
$$

\section{STABILITY ANALYSIS}

\subsection{FGM cylindrical panel under uniform external pressure in thermal environment}

The FGM cylindrical panel with tangentially restrained edges is assumed to be exposed to uniform thermal environment and simultaneously subjected to external pressure $q$ uniformly distributed on the outer surface of the panel. Introduction of Eq. (33) and Eqs. (28), (29) into Eq. (24) result in the following expression

$$
q=b_{11} \bar{W}+b_{21} \bar{W}(\bar{W}+\mu)+b_{31} \bar{W}(\bar{W}+2 \mu)+b_{41} \bar{W}(\bar{W}+\mu)(\bar{W}+2 \mu)-b_{51} \Delta T,
$$

where

$$
\begin{aligned}
& b_{11}=-\frac{\left(\bar{D}_{2} \bar{D}_{5}-\bar{D}_{3} \bar{D}_{4}\right)}{9 \bar{D}_{6} B_{h}^{6}} m n \pi^{8}\left(m^{2} B_{a}^{2}+n^{2}\right)^{3}+\frac{m n \pi^{6}}{16 B_{h}^{4}}\left(\frac{4}{3} \bar{D}_{2}+\bar{D}_{4}\right)\left(m^{2} B_{a}^{2}+n^{2}\right)^{2} \\
& +\frac{\bar{E}_{1} m^{5} n \pi^{2} B_{a}^{6} R_{a}^{2}}{16 B_{h}^{2}\left(m^{2} B_{a}^{2}+n^{2}\right)^{2}} \bar{\xi}_{m n}-\frac{B_{a} R_{a}}{B_{h}} e_{21}+\frac{m n \pi^{2} \bar{D}_{1} B_{a}^{2}}{16 B_{h}^{4}} \bar{\xi}_{m n}\left[B_{a}^{2} K_{1}+\pi^{2}\left(m^{2} B_{a}^{2}+n^{2}\right) K_{2}\right], \\
& b_{21}=\frac{m n \pi^{4}}{16 B_{h}^{2}} \bar{\xi}_{m n}\left(m^{2} B_{a}^{2} e_{11}+n^{2} e_{21}\right)-\frac{2 m^{4} n^{2} \pi^{2} \bar{E}_{1} B_{a}^{5} R_{a}}{3 B_{h}^{3}\left(m^{2} B_{a}^{2}+n^{2}\right)^{2}}\left(2 \bar{\xi}_{m n}-1\right), \\
& b_{31}=-\frac{\bar{E}_{1} n^{2} \pi^{2} B_{a} R_{a}}{24 B_{h}^{3}}\left[\frac{4 \pi^{2} m^{2} B_{a}^{2}}{3 \bar{D}_{6} B_{h}^{2}}\left(3 \bar{D}_{4}-4 \bar{D}_{5}\right)+1\right]-\frac{B_{a} R_{a}}{B_{h}} e_{22}, \\
& b_{41}=\frac{\bar{E}_{1} m n \pi^{6}}{256 B_{h}^{4}}\left(m^{4} B_{a}^{4}+n^{4}\right) \bar{\xi}_{m n}+\frac{m n \pi^{4}}{16 B_{h}^{2}} \bar{\xi}_{m n}\left(m^{2} B_{a}^{2} e_{12}+n^{2} e_{22}\right), \\
& b_{51}=H(T)\left[\frac{m n \pi^{4}}{16 B_{h}^{2}} \bar{\xi}_{m n}\left(m^{2} B_{a}^{2} e_{13}+n^{2} e_{23}\right)(\bar{W}+\mu)-\frac{B_{a} R_{a}}{B_{h}} e_{23}\right] .
\end{aligned}
$$

\subsection{FGM cylindrical panel under uniform temperature rise}

Tangentially restrained edge thick FGM cylindrical panel is rested on an elastic foundation and exposed to uniformly elevated temperature field in absence of external 
pressure, i.e. $q=0$. From Eq. (34), relation of temperature-deflection curves can be expressed as

$$
\Delta T=\frac{1}{b_{51}}\left[b_{11} \bar{W}+b_{21} \bar{W}(\bar{W}+\mu)+b_{31} \bar{W}(\bar{W}+2 \mu)+b_{41} \bar{W}(\bar{W}+\mu)(\bar{W}+2 \mu)\right] .
$$

Eq. (36) indicates that FGM cylindrical panels under bending at the onset of thermal loading and temperature-deflection curves have no bifurcation point in general. However, thermally loaded FGM cylindrical panels may exhibit a bifurcation type response in some particular cases. Specifically, seeing expression of $b_{51}$, bifurcation type buckling behavior may be predicted for FGM cylindrical panels of which geometry and material parameters and tangential edge constraints satisfy condition

$$
\frac{m n \pi^{4}}{16 B_{h}^{2}} \bar{\zeta}_{m n}\left(m^{2} B_{a}^{2} e_{13}+n^{2} e_{23}\right) \mu-\frac{B_{a} R_{a}}{B_{h}} e_{23}=0 .
$$

Eq. (37) shows that geometrically perfect FGM cylindrical panels, i.e. $\mu=0, R_{a} \neq$ 0 , only buckle as $e_{23}=0$ implying $s_{2}=0$ and straight edges $y=0, b$ are freely movable. Also, bifurcation buckling behavior only occurs for imperfect cylindrical panels with tangential edge restraints as the imperfection size $\mu$ satisfy condition

$$
\mu=\frac{16 e_{23} B_{a} B_{h} R_{a}}{m n \pi^{4} \bar{\xi}_{m n}\left(m^{2} B_{a}^{2} e_{13}+n^{2} e_{23}\right)} .
$$

Eq. (36) is explicit expression of temperature-deflection relation for FGM cylindrical panels in case of material properties are temperature independent. In contrast, as temperature dependence of material properties is included, Eq. (36) is implicit expression and an iteration algorithm must be utilized to obtain temperature-deflection curves. Detailed procedure of iteration process is similar as that suggested in the work [20] and is omitted here for sake of brevity. The error tolerance of iteration of the present study is 0.001 .

\subsection{FGM cylindrical panel under uniform axial compression}

Consider a thick FGM cylindrical panel resting on an elastic foundation and exposed to thermal environment in the absence of external pressure. The panel is subjected to axial compressive load $F$ uniformly distributed on two curved edges $x=0, a$ assumed to be freely movable, whereas two unloaded straight edges $y=0, b$ are tangentially restrained. In this case, $\bar{N}_{x 0}=-F[4,19]$ and $\bar{N}_{y 0}$ is determined by following the same procedure described in the previous section as

$$
\bar{N}_{y 0}=e_{31} \bar{N}_{x 0}+e_{32} \bar{W}+e_{33} \bar{W}(\bar{W}+2 \mu)-e_{34} H(T) \Delta T,
$$

where

$$
\begin{aligned}
& e_{34}=\frac{\bar{s}_{2}}{\bar{E}_{1}+\bar{s}_{2}}, \quad e_{31}=v e_{34}, \quad e_{33}=\frac{n^{2} \pi^{2} \bar{E}_{1}}{8 B_{h}^{2}} e_{34} \\
& e_{32}=e_{34}\left[\frac{4 \bar{E}_{1} m B_{a}^{3} R_{a}\left(m^{2} B_{a}^{2}-v n^{2}\right)}{n \pi^{2} B_{h}\left(m^{2} B_{a}^{2}+n^{2}\right)^{2}}+\frac{16 \bar{E}_{4}}{3 m \pi B_{h}}\left(\bar{B}_{2}+\frac{n \pi}{B_{h}}\right)-\frac{4 \bar{E}_{2} \bar{B}_{2}}{m \pi B_{h}}-\frac{4 \bar{E}_{1} B_{a} R_{a}}{m n \pi^{2} B_{h}}\right] .
\end{aligned}
$$


Substitution of $\bar{N}_{x 0}, \bar{N}_{y 0}$ into Eq. (24) leads to the following expression

$$
\begin{aligned}
F= & \frac{1}{\bar{A}}\left[b_{12} \bar{W}+b_{22} \bar{W}(\bar{W}+\mu)+b_{32} \bar{W}(\bar{W}+2 \mu)\right. \\
& \left.+b_{42} \bar{W}(\bar{W}+\mu)(\bar{W}+2 \mu)-b_{52} H(T) \Delta(T)\right],
\end{aligned}
$$

where

$$
\begin{aligned}
b_{12}= & -\frac{\left(\bar{D}_{2} \bar{D}_{5}-\bar{D}_{3} \bar{D}_{4}\right)}{9 \bar{D}_{6} B_{h}^{6}} m n \pi^{8}\left(m^{2} B_{a}^{2}+n^{2}\right)^{3} \\
& +\frac{m n \pi^{6}}{16 B_{h}^{4}}\left(\frac{4}{3} \bar{D}_{2}+\bar{D}_{4}\right)\left(m^{2} B_{a}^{2}+n^{2}\right)^{2}+\frac{\bar{E}_{1} m^{5} n \pi^{2} B_{a}^{6} R_{a}^{2}}{16 B_{h}^{2}\left(m^{2} B_{a}^{2}+n^{2}\right)^{2}} \bar{\xi}_{m n} \\
& +\frac{m n \pi^{2} \bar{D}_{1} B_{a}^{2}}{16 B_{h}^{4}} \bar{\xi}_{m n}\left[B_{a}^{2} K_{1}+\pi^{2}\left(m^{2} B_{a}^{2}+n^{2}\right) K_{2}\right]-\frac{B_{a} R_{a} e_{32}}{B_{h}}, \\
b_{22}= & \frac{m n \pi^{4}}{16 B_{h}^{2}} \bar{\xi}_{m n} n^{2} e_{32}-\frac{2 m^{4} n^{2} \pi^{2} \bar{E}_{1} B_{a}^{5} R_{a}}{3 B_{h}^{3}\left(m^{2} B_{a}^{2}+n^{2}\right)^{2}}\left(2 \bar{\xi}_{m n}-1\right), \\
b_{32}= & -\frac{\bar{E}_{1} n^{2} \pi^{2} B_{a} R_{a}}{24 B_{h}^{3}}\left[\frac{4 \pi^{2} m^{2} B_{a}^{2}}{3 \bar{D}_{6} B_{h}^{2}}\left(3 \bar{D}_{4}-4 \bar{D}_{5}\right)+1\right]-\frac{B_{a} R_{a}}{B_{h}} e_{33}, \\
b_{42}= & \frac{\bar{E}_{1} m n \pi^{6}}{256 B_{h}^{4}}\left(m^{4} B_{a}^{4}+n^{4}\right) \bar{\xi}_{m n}+\frac{m n \pi^{4}}{16 B_{h}^{2}} \bar{\xi}_{m n} n^{2} e_{33}, \\
b_{52}= & \frac{m n \pi^{4}}{16 B_{h}^{2}} \bar{\xi}_{m n} n^{2} e_{34}(\bar{W}+\mu)-\frac{B_{a} R_{a}}{B_{h}} e_{34}, \\
\bar{A}= & \frac{m n \pi^{4}}{16 B_{h}^{2}} \bar{\xi}_{m n}\left(m^{2} B_{a}^{2}+n^{2} e_{31}\right)(\bar{W}+\mu)-\frac{B_{a} R_{a}}{B_{h}} e_{31} .
\end{aligned}
$$

It is observed from Eqs. (41) and (42) that axially loaded FGM cylindrical panels will exhibit a bifurcation type buckling behavior as

$$
\frac{m n \pi^{4}}{16 B_{h}^{2}} \bar{\xi}_{m n}\left(m^{2} B_{a}^{2}+n^{2} e_{31}\right) \mu-\frac{B_{a} R_{a}}{B_{h}} e_{31}=0,
$$

with corresponding buckling compressive load is predicted as

$$
F_{b}=\frac{16 B_{h}^{2}}{m n \pi^{4} \bar{\xi}_{m n}\left(m^{2} B_{a}^{2}+n^{2} e_{31}\right)} b_{12} .
$$

Eq. (43) indicates that, under compression, perfect FGM cylindrical panel (i.e. $R_{a} \neq 0, \mu=0$ ) only buckle as $e_{31}=0$ deducing the average tangential stiffness $s_{2}=0$ and two straight edges $y=0, b$ are freely movable. In contrast, imperfect FGM cylindrical panel subjected to axial compression will experience bifurcation type buckling at tangential stiffness $\bar{s}_{2}^{b}$ predicted from Eqs. (41) and (42) as

$$
\bar{s}_{2}^{b}=\frac{\bar{E}_{1} m^{3} n \pi^{4} \bar{\xi}_{m n} B_{a}^{2} \mu}{16 v B_{a} R_{a} B_{h}-m n \pi^{4} \bar{\xi}_{m n} \mu\left(m^{2} B_{a}^{2}+v n^{2}\right)} .
$$


To measure the degree of edge constraint in a more convenient way, alternate tangential stiffness parameters $\lambda_{1}$ and $\lambda_{2}$ are introduced such that $\lambda_{1}=0$ and $\lambda_{1}=1$ correspond to movable and immovable edges at $x=0$ and $a$, respectively. Similarly, $\lambda_{2}=0$ and $\lambda_{2}=1$ correspond to movable and immovable edges at $y=0$ and $b$, respectively. Partially restrained edges at $x=0, a$ and $y=0, b$ are defined by $0<\lambda_{1}<1$ and $0<\lambda_{2}<1$, respectively. In the present study, these alternate tangential stiffness parameters are defined by

$$
\lambda_{1}=\frac{\bar{s}_{1}}{\bar{E}_{1}\left(T_{0}\right)+\bar{s}_{1}}, \quad \lambda_{2}=\frac{\bar{s}_{2}}{\bar{E}_{1}\left(T_{0}\right)+\bar{s}_{2}},
$$

in which $\bar{E}_{1}\left(T_{0}\right)$ is value of $\bar{E}_{1}$ calculated at room temperature $T_{0}$.

\section{RESULTS AND DISCUSSION}

This section presents numerical results for square plan-form panels $(a=b)$ made of functionally graded materials and for deformation mode with half wave numbers $m=n=1$.

To validate the proposed approach, nonlinear response of perfect FGM cylindrical panels with movable edges $\left(\lambda_{1}=\lambda_{2}=0\right)$, without elastic foundations $\left(K_{1}=K_{2}=\right.$ $0)$ and subjected to uniform external pressure is considered. The cylindrical panel has $a=b=0.2 \mathrm{~m}, R=1.0 \mathrm{~m}, h=0.01 \mathrm{~m}$ and is made of Aluminum (Al) and Zirconia $\left(\mathrm{ZrO}_{2}\right)$. The temperature independent material properties are $E_{m}=70 \mathrm{GPa}, E_{c}=151$ GPa and $v=0.3$ for both constituents.

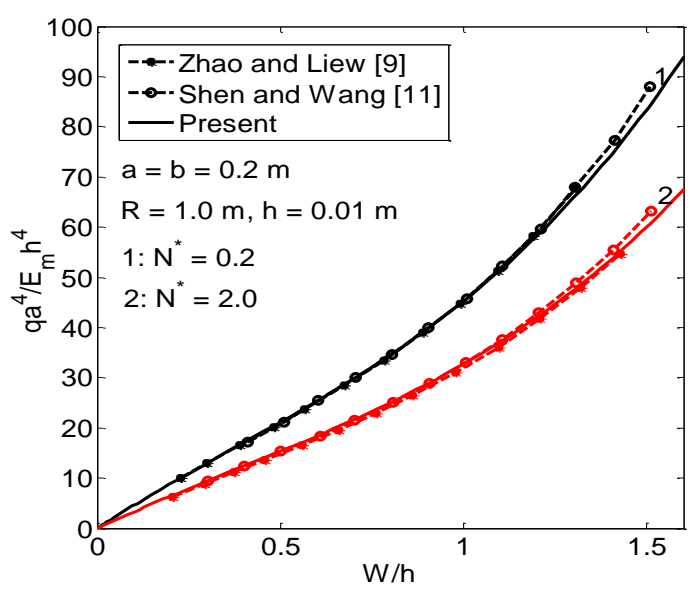

Fig. 2. Comparisons of pressure-deflection curves for $\mathrm{ZrO}_{2} / \mathrm{Al}$ cylindrical panels

The load-deflection curves for a simply supported $\mathrm{ZrO}_{2} / \mathrm{Al}$ cylindrical panel are plotted in Fig. 2 and compared with results of Zhao and Liew [9] using the element-free kp-Ritz method and with results of Shen and Wang [11] utilizing a two-step perturbation technique. In the Fig. 2, $N^{*}$ is volume fraction index as the definition in Eq. (1) is given 
for case of ceramic and metal constituents are interchanged, i.e. $V_{c}(z)=(1 / 2+z / h)^{N^{*}}$. It is evident that a very good agreement is obtained in this comparison.

The remainder of this section presents numerical results for FGM cylindrical panels composed of silicon nitride $\left(\mathrm{Si}_{3} \mathrm{~N}_{4}\right)$ and stainless steel (SUS304). The material properties Pr, such as elasticity modulus $E$ and thermal expansion coefficient $\alpha$ can be expressed as a nonlinear function of temperature [22]

$$
\operatorname{Pr}=P_{0}\left(P_{-1} T^{-1}+1+P_{1} T+P_{2} T^{2}+P_{3} T^{3}\right),
$$

in which $T=T_{0}+\Delta T$ and $T_{0}=300 \mathrm{~K}$ (room temperature), $P_{0}, P_{-1}, P_{1}, P_{2}$ and $P_{3}$ are the coefficients of temperature $T(\mathrm{~K})$ and are unique to the constituent materials. Specific values of these coefficients for $E$ and $\alpha$ of silicon nitride and stainless steel are given by Reddy and Chin [23] and are listed in Tab. 1. Poisson's ratio is assumed to be a constant $v=0.3$. In addition, temperature-dependent and temperature-independent material properties will be written as T-D and T-ID, respectively, for sake of brevity. The T-ID are material properties calculated at room temperature $T_{0}=300 \mathrm{~K}$. In characterizing the behavior of the panels, deformations in which the central region of a panel moves towards the plane that contains the four corners of the panel are referred to as inward deflections, i.e. positive deflection. Deformations in the opposite direction are referred to as outward deflection, i.e. negative deflection.

Table 1. Temperature-dependent thermo-elastic coefficients for silicon nitride and stainless steel (Reddy and Chin [23])

\begin{tabular}{|c|c|c|c|c|c|c|}
\hline Materials & Properties & $P_{0}$ & $P_{-1}$ & $P_{1}$ & $P_{2}$ & $P_{3}$ \\
\hline Silicon nitride & $E(\mathrm{~Pa})$ & $348.43 \mathrm{e}+9$ & 0 & $-3.070 \mathrm{e}-4$ & $2.160 \mathrm{e}-7$ & $-8.946 \mathrm{e}-11$ \\
\cline { 2 - 7 } & $\alpha(1 / \mathrm{K})$ & $5.8723 \mathrm{e}-6$ & 0 & $9.095 \mathrm{e}-4$ & 0 & 0 \\
\hline \multirow{3}{*}{ Stainless steel } & $E(\mathrm{~Pa})$ & $201.04 \mathrm{e}+9$ & 0 & $3.079 \mathrm{e}-4$ & $-6.534 \mathrm{e}-7$ & 0 \\
\cline { 2 - 7 } & $\alpha(1 / \mathrm{K})$ & $12.330 \mathrm{e}-6$ & 0 & $8.086 \mathrm{e}-4$ & 0 & 0 \\
\hline
\end{tabular}

Fig. 3 analyzes the effects of parameters $\lambda_{1}, \lambda_{2}$ on the nonlinear response of FGM cylindrical panels supported by an elastic foundation and exposed to a thermal environment. As can be seen, FGM cylindrical panels tangentially restrained at all edges have no bifurcation behavior and are monotonically deflected towards convex side. In contrast, Fig. 4, plotted as counterparts of the Fig. 3 for case of $\lambda_{2}=0$, shows that FGM cylindrical panels with two freely movable straight edges experience bifurcation type buckling and relatively benign postbuckling curves. These figures indicate sensitive influences of tangential constraints of straight edges on the deflection shape of the cylindrical panels and that the difference between temperature-deflection curves for two T-D and T-ID cases become bigger for partially restrained edge FGM cylindrical panels in comparison with immovable edge counterparts.

Fig. 5 assesses the effects of power index $N$ and stiffness of elastic foundation on the mechanical postbuckling of axially compressed FGM cylindrical panels with all movable edges. As expected, buckling loads and postbuckling curves are enhanced due to increase 


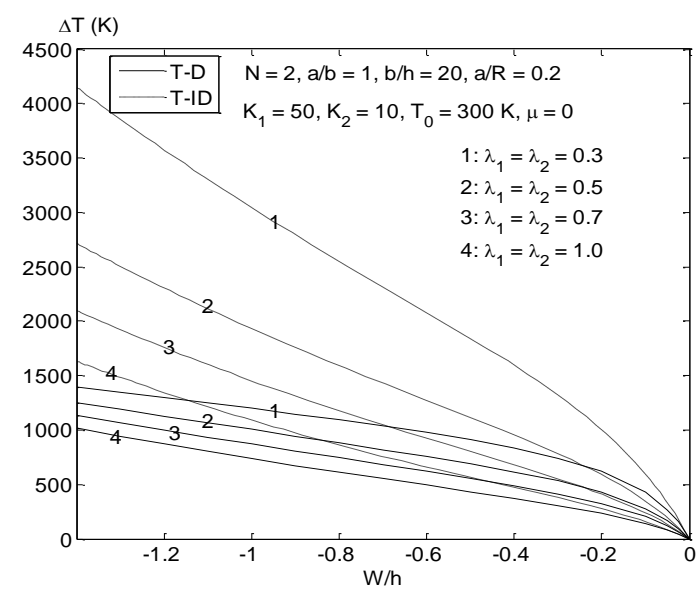

Fig. 3. Effects of tangential edge constraint on temperature-deflection curves of perfect FGM cylindrical panels

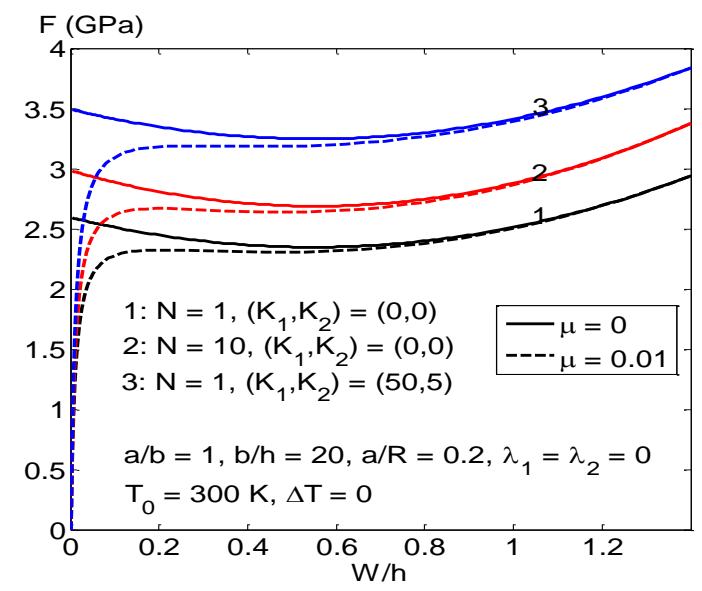

Fig. 5. Effects of the index $N$ and elastic foundations on the postbuckling of FGM panel under axial compression

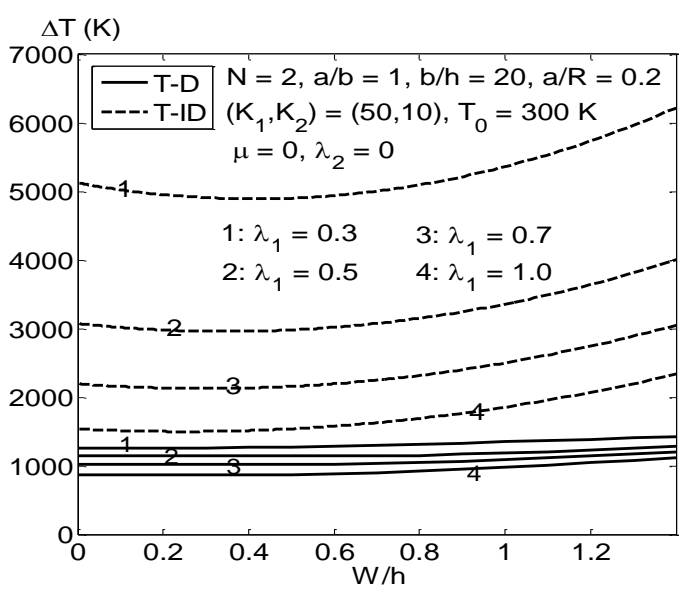

Fig. 4. Counterparts of the Fig. 3 for case of $\lambda_{2}=0$

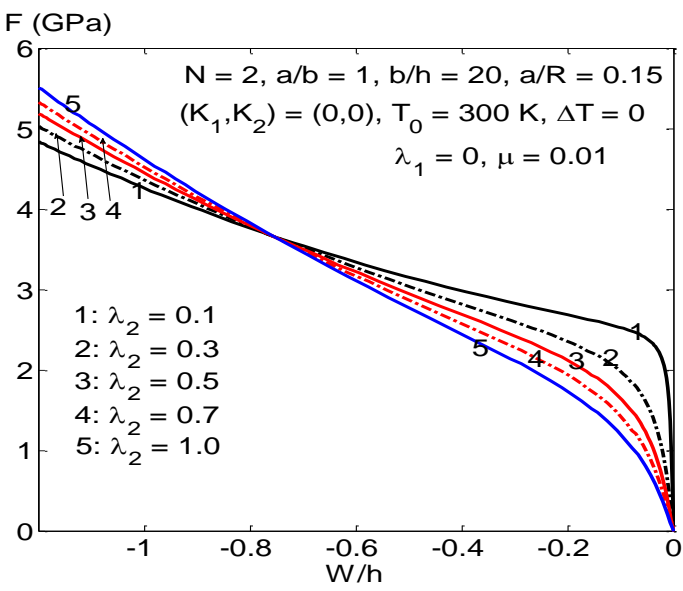

Fig. 6. Effects of parameter $\lambda_{2}$ on the nonlinear response of imperfect FGM cylindrical panels under axial compression

in stiffness of foundation and/or percentage of ceramic constituent in FGM cylindrical panel.

The effects of imperfection and in-plane constraint of straight edges $y=0, b$ on the nonlinear response of axially compressed FGM cylindrical panels without foundation interaction and thermal environment are analyzed in Figs. 6 and 7. The Fig. 6, plotted with $\mu=0.01$ and five various values of parameter $\lambda_{2}$, shows that the axially compressed FGM cylindrical panels are deflected towards convex side with no bifurcation buckling. Moreover, load-deflection equilibrium paths are dropped as $\lambda_{2}$ increases. It seems that constraint of straight edges produces compressive stress and make the curvature of the 
cylindrical panels is increased and, as a result, the cylindrical panels are deflected outwards under axial compression.

Conversely, the panel will be deflected inwards and capability of compressive load carrying is enhanced due to increase in $\lambda_{2}$ for higher value of imperfection size $\mu$ as shown in the Fig. 7 depicted for $\mu=0.1$. Especially, the axially compressed FGM cylindrical panel will exhibit a bifurcation type buckling behavior as degree of tangential constraint $\lambda_{2}$ reaches a definite value as predicted by Eqs. (45) and (46).

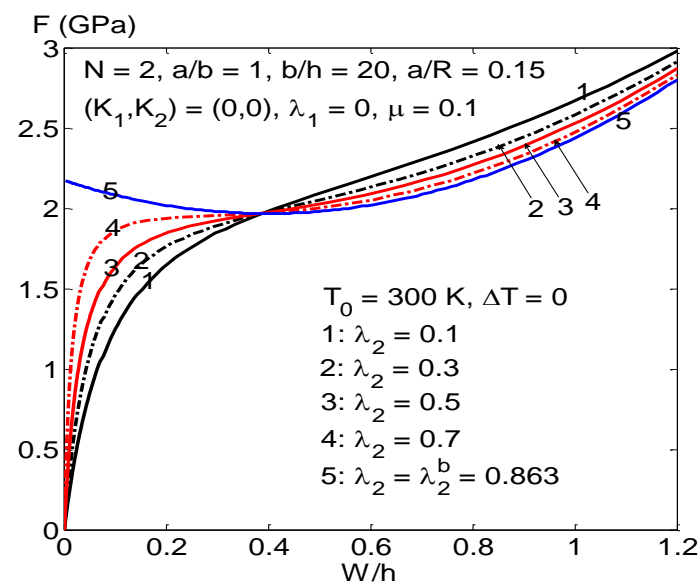

Fig. 7. Counterparts of the Fig. 6 for case of $\mu=0.1$ and $\lambda_{2}=\lambda_{2}^{b}$

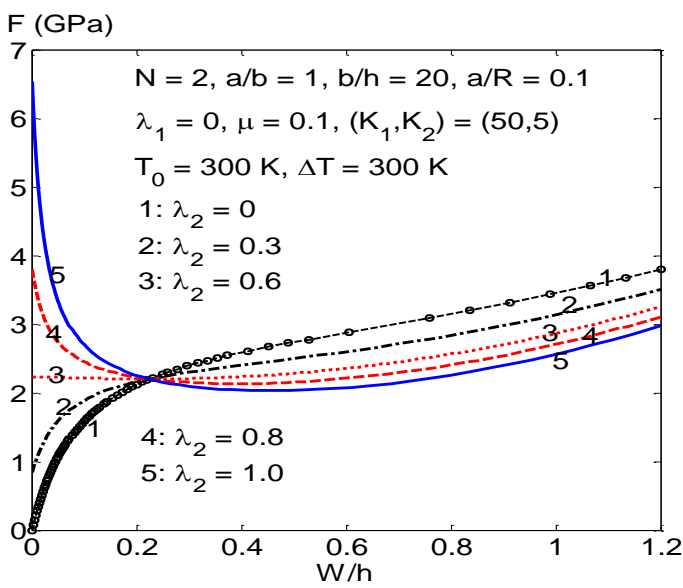

Fig. 8. Effects of parameter $\lambda_{2}$ and thermal environment on the thermomechanical postbuckling of FGM cylindrical panels

The effects of parameter $\lambda_{2}$ and thermal environment $(\Delta T=300 \mathrm{~K})$ on the thermomechanical postbuckling of imperfect FGM cylindrical panels subjected to uniform axial compression and resting on an elastic foundation are illustrated in Fig. 8. As can be observed, imperfect FGM cylindrical panels experience a bifurcation type buckling due to presence of elevated temperature and constraint of straight edges, i.e. $\Delta T \neq 0$ and $\lambda_{2} \neq 0$, and bifurcation point compressive loads are enhanced by virtue of increasing in $\lambda_{2}$. Furthermore, a snap-through phenomenon with severe intensity occurs for FGM cylindrical panels rigorously restrained in tangential motion of straight edges, i.e. higher values of $\lambda_{2}$.

Finally, the effects of tangential edge constraints on the thermomechanical nonlinear response of pressure-loaded FGM cylindrical panels exposed to thermal environment without and with foundation interaction are analyzed in Figs. 9 and 10, respectively. It is evident that tangentially restrained edges panel will exhibit a bifurcation buckling behavior as exposed to thermal environment $(\Delta T=200 \mathrm{~K})$, and both bifurcation point pressure and the severity of snap-through instability are considerably enhanced as parameters $\lambda_{1}, \lambda_{2}$ are increased. In addition, pressure-deflection curves are higher and more stable due to the presence of an elastic foundation, although bifurcation buckling pressures, predicted by final term at right hand side of Eq. (34), are unchanged. 


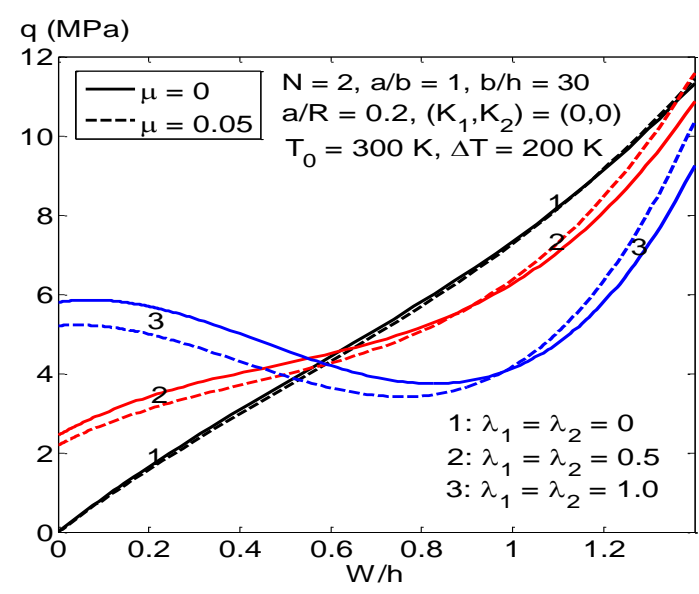

Fig. 9. Effects of edge constraints on the nonlinear response of pressure-loaded FGM cylindrical panels in thermal environment

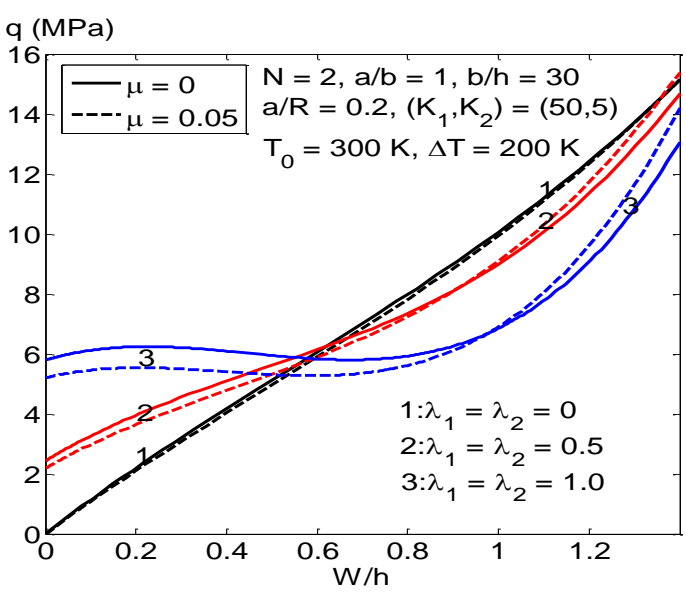

Fig. 10. Counterparts of the Fig. 9 for case of $K_{1}=50, K_{2}=5$

\section{CONCLUDING REMARKS}

The nonlinear response and postbuckling behavior of thick FGM cylindrical panels subjected to thermal, mechanical and thermomechanical loadings have been analyzed. Formulations are based on the higher order shear deformation shell theory taking geometrical nonlinearity, initial imperfection, panel-foundation interaction, tangential edge constraints and temperature dependent material properties into consideration. The analysis reveals that the tangential constraints of boundary edges, especially straight edges, have extremely sensitive influences on the nonlinear response and postbuckling of FGM cylindrical panels, especially for case of these panels subjected to thermal loading and axial compression. The results also show that temperature dependence of material properties has deteriorative and pronounced effects on the thermal buckling and postbuckling behavior of thick FGM cylindrical panels resting on elastic foundations and with tangentially restrained edges. Furthermore, elastic foundations, especially Pasternak type foundations, have beneficial influences on the nonlinear stability of FGM panels on both aspects are that increase in load carrying capacity and decrease in the severity of snapthrough instability.

\section{ACKNOWLEDGEMENT}

This research is funded by Vietnam National Foundation for Science and Technology Development (NAFOSTED) under grant number 107.02-2014.09.

\section{REFERENCES}

[1] J. Woo, S. A. Meguid, J. C. Stranart, and K. M. Liew. Thermomechanical postbuckling analysis of moderately thick functionally graded plates and shallow shells. International Journal of Mechanical Sciences, 47, (8), (2005), pp. 1147-1171. 
[2] N. D. Duc and H. V. Tung. Nonlinear response of pressure-loaded functionally graded cylindrical panels with temperature effects. Composite Structures, 92, (7), (2010), pp. 1664-1672.

[3] N. D. Duc and H. V. Tung. Nonlinear analysis of stability for functionally graded cylindrical panels under axial compression. Computational Materials Science, 49, (4), (2010), pp. S313S316.

[4] H. V. Tung and N. D. Duc. Nonlinear response of shear deformable FGM curved panels resting on elastic foundations and subjected to mechanical and thermal loading conditions. Applied Mathematical Modelling, 38, (11), (2014), pp. 2848-2866.

[5] H. S. Shen. Postbuckling analysis of axially loaded functionally graded cylindrical panels in thermal environments. International Journal of Solids and Structures, 39, (24), (2002), pp. 59916010.

[6] H. S. Shen and A. Y. T. Leung. Postbuckling of pressure-loaded functionally graded cylindrical panels in thermal environments. Journal of Engineering Mechanics, 129, (4), (2003), pp. 414425.

[7] H. S. Shen and K. M. Liew. Postbuckling of axially loaded functionally graded cylindrical panels with piezoelectric actuators in thermal environments. Journal of Engineering Mechanics, 130, (8), (2004), pp. 982-995.

[8] J. Yang, K. M. Liew, Y. F. Wu, and S. Kitipornchai. Thermo-mechanical post-buckling of FGM cylindrical panels with temperature-dependent properties. International Journal of Solids and Structures, 43, (2), (2006), pp. 307-324.

[9] X. Zhao and K. M. Liew. Geometrically nonlinear analysis of functionally graded shells. International Journal of Mechanical Sciences, 51, (2), (2009), pp. 131-144.

[10] K. M. Liew, X. Zhao, and Y. Y. Lee. Postbuckling responses of functionally graded cylindrical shells under axial compression and thermal loads. Composites Part B: Engineering, 43, (3), (2012), pp. 1621-1630.

[11] H. S. Shen and H. Wang. Nonlinear bending of FGM cylindrical panels resting on elastic foundations in thermal environments. European Journal of Mechanics-A/Solids, 49, (2015), pp. 49-59.

[12] H. S. Shen and H. Wang. Nonlinear bending and postbuckling of FGM cylindrical panels subjected to combined loadings and resting on elastic foundations in thermal environments. Composites Part B: Engineering, 78, (2015), pp. 202-213.

[13] D. H. Bich, D. V. Dung, and V. H. Nam. Nonlinear dynamical analysis of eccentrically stiffened functionally graded cylindrical panels. Composite Structures, 94, (8), (2012), pp. 24652473.

[14] D. H. Bich, D. V. Dung, and V. H. Nam. Nonlinear dynamic analysis of eccentrically stiffened imperfect functionally graded doubly curved thin shallow shells. Composite Structures, 96, (2013), pp. 384-395.

[15] N. D. Duc and T. Q. Quan. Nonlinear postbuckling of imperfect eccentrically stiffened PFGM double curved thin shallow shells on elastic foundations in thermal environments. Composite Structures, 106, (2013), pp. 590-600.

[16] N. D. Duc and T. Q. Quan. Nonlinear response of imperfect eccentrically stiffened FGM cylindrical panels on elastic foundation subjected to mechanical loads. European Journal of Mechanics-A/Solids, 46, (2014), pp. 60-71.

[17] L. Librescu, W. Lin, M. P. Nemeth, and J. H. Starnes Jr. Thermomechanical postbuckling of geometrically imperfect flat and curved panels taking into account tangential edge constraints. Journal of Thermal Stresses, 18, (4), (1995), pp. 465-482. 
[18] L. Librescu and W. Lin. Vibration of thermomechanically loaded flat and curved panels taking into account geometric imperfections and tangential edge restraints. International Journal of Solids and Structures, 34, (17), (1997), pp. 2161-2181.

[19] H. V. Tung. Postbuckling behavior of functionally graded cylindrical panels with tangential edge constraints and resting on elastic foundations. Composite Structures, 100, (2013), pp. 532 541.

[20] H. V. Tung. Postbuckling of functionally graded cylindrical shells with tangential edge restraints and temperature-dependent properties. Acta Mechanica, 225, (6), (2014), pp. 17951808.

[21] J. N. Reddy and C. F. Liu. A higher-order shear deformation theory of laminated elastic shells. International Journal of Engineering Science, 23, (3), (1985), pp. 319-330.

[22] Y. S. Touloukian. Thermophysical properties of high temperature solid materials. New York: MacMillan, (1967).

[23] J. N. Reddy and C. D. Chin. Thermomechanical analysis of functionally graded cylinders and plates. Journal of Thermal Stresses, 21, (6), (1998), pp. 593-626. 